\title{
Role of Intra-Operative Nerve Monitoring in Thyroidectomies: An Institutional Review
}

\author{
Naren N. Venkatesan*, Sharon H. Gnagi, Michael P. Underbrink \\ Department of Otolaryngology-Head and Neck Surgery, University of Texas-Medical Branch at Galveston \\ School of Medicine, Galveston, USA \\ Email: ${ }^{*}$ narennv@gmail.com
}

Received 14 April 2014; revised 13 May 2014; accepted 12 June 2014

Copyright (C) 2014 by authors and Scientific Research Publishing Inc.

This work is licensed under the Creative Commons Attribution International License (CC BY). http://creativecommons.org/licenses/by/4.0/

cC) (7) Open Access

\begin{abstract}
Injury to the Recurrent Laryngeal Nerve (RLN) is a worrisome complication of a thyroidectomy. Intra-operative nerve monitoring (IONM) of the RLN has gained prevalence as an aid to prevent injury. We reviewed our series and other studies in literature for insight. A chart review was carried out to identify all patients who underwent a thyroidectomy between 2005 and 2010 . IONM was implemented by the Otolaryngology service in 2007. All identified patients were separated into three groups: 1) Otolaryngology service with IONM, 2) Otolaryngology service without IONM, and 3) General Surgery service without IONM. Several factors were noted, including age, sex, thyroid disease, extent of thyroidectomy, and RLN injury along with recovery. 230 patients underwent thyroidectomy from 2005-2010. 60 patients were isolated in the IONM-Otolaryngology group with 3 patients suffering injury. 109 patients underwent a thyroidectomy by the Otolaryngology service without IONM with 8 patients suffering nerve damage. In the third group, 61 patients underwent a thyroidectomy by General Surgery without IONM with 4 patients suffering damage. Of the thyroid pathology, 10 patients had Multinodular Goiter while 4 had Papillary Cancer and 1 had a Follicular Adenoma. The most severe complication of a thyroidectomy is RLN injury. In order to further decrease the risk of RLN injury, IONM has been employed. From our review and other studies, there does not appear to be a significant difference in rates of RLN injury with or without use of nerve monitoring. An interesting note is the increased prevalence of nerve injuries in Multinodular Goiter-a finding that merits further study to evaluate the role of IONM.
\end{abstract}

\section{Keywords}

Recurrent Laryngeal Nerve Injruy, Vocal Cord Paralysis, Thyroidectomy, Intra-Operative Nerve Monitoring

\footnotetext{
${ }^{*}$ Corresponding author.
} 


\section{Introduction}

A thyroidectomy is a routine procedure performed by Otolaryngologists, General Surgeons, and Endocrine Surgeons. The surgery is performed most commonly for either a Goiter causing compressive symptoms or a Nodule confirmed or suspicious for cancer. While fairly routine, this operation carries two significant risks: damage to the recurrent laryngeal nerve and/or damage to the parathyroids. Damage to the recurrent laryngeal nerve greatly affects the patient's voice, and in rare bilateral cases, may mandate the need for a tracheostomy. The obvious decreased social and psychological functioning after such an injury cannot be underestimated. Therefore, the importance of preventing this complication has not been lost on the surgeon.

In order to better preserve the Recurrent Laryngeal Nerve (RLN) during a thyroidectomy, Lahey and Riddell advocated routine nerve dissection and visualization [1] [2]. As more data have been collected, this practice has now become the gold standard of care in thyroidectomies to identify the RLN [3]-[5]. Despite the incorporation of routine identification, RLN injury during surgery or noted post-operatively continues to occur. While these rates have decreased due to this practice, large studies still show $0.4 \%$ to $1 \%$ risk of injury [6].

As the impact of RLN injury is tremendous on a patient's daily function, any attempt to help decrease those rates would seem to be beneficial. Intra-operative nerve monitoring (IONM) has gained favor amongst surgeons, especially in the last decade. Three reasons are typically given for IONM-initial identification of the RLN, aid in dissection after initial identification of the RLN, and assessment after removal of the thyroid to ensure patency of the RLN [7]. To that end, several recent studies have been performed comparing the rate of RLN injury in cases with and without IONM. Barczynski demonstrated that the use of IONM helped decrease the rate of transient paralysis when compared to visual identification alone by a statistically significant difference of $2.9 \%$ [8].

However, other work has demonstrated that the benefit of IONM may not be as notable. Further research regarding the statistical power generated by studies comparing IONM with only visual identification suggests that several million patients are needed to show a difference when only discussing multinodular goiter and nearly 40,000 patients when discussing only thyroid cancer [3]. With such high patient numbers, the actual benefit of IONM in a thyroidectomy for each individual patient may be minimal. A further challenge to assessing the efficacy of IONM is the various systems currently in use. Intra-operative nerve monitoring systems currently exist which function either through direct placement of needle electrodes-either transcricothyroid or intralaryngealor surface electrodes via endotracheal tube or post-cricoid. The different systems make it difficult to discern the value of IONM, and only recently have prospective studies been undertaken to address this practice. Assessing the benefit of IONM is an integral aspect of the management of patients undergoing thyroidectomy for two reasons. If it provides a significant decrease in the risk of RLN injury, then it needs to become a standard of practice. Second, the price of the IONM, while minimal in comparison to the total cost of the procedure and hospital stay, may be avoided. To that end, we provide a retrospective review of thyroidectomies performed at our institution, the University of Texas Medical Branch at Galveston, comparing the data of patients in whom the IONM was and was not used.

\section{Methods}

A retrospective chart review of all patients undergoing a partial or total thyroidectomy from 2005-2010 was carried out. The study was approved by the institutional review board. The charts of patients were obtained through the medical records department based on billing codes. Patients known to have preoperative vocal cord dysfunction, paresis, or paralysis and those undergoing laryngectomy or intentional nerve sacrifice due to tumor adherence or involvement were excluded from analysis. Thyroidectomies performed by both the General Surgery service and Otolaryngology service were included in the study. Intra-Operative Nerve Monitoring began initially at this institution in 2007, and by the end of 2008, its use was routine amongst all Otolaryngologists in our department. The Medtronic (Minneapolis, MN, USA) NIM EMG endotracheal tube 3.0 version is used at our institution. A brief lapse in operations occurred in 2008 due to a national disaster. Unmonitored cases were performed by all surgeons prior to 2007 and by the General Surgery service even after the introduction of the Intra-Operative Nerve Monitoring System.

Operative notes were obtained for each patient and were reviewed by the authors for operative details, use of nerve monitoring, identification of the recurrent laryngeal nerve, and any noted aberrant anatomy. Age, gender, disease of the thyroid, concurrent neck surgery, and previous neck operations were also noted. Post-operative paresis and paralysis diagnosed by flexible laryngoscopy were recorded. As many patients suffer from tempo- 
rary hoarseness following intubation, only conclusive evidence of paresis or paralysis was recorded as an occurrence of nerve injury. The data was then analyzed for incidence of injury by looking at the distribution of nerve injury across 3 groups - thyroidectomies performed by Otolaryngologists with IONM, thyroidetomies performed by Otolaryngologists without IONM, and thyroidectomies performed by General Surgeons without IONM. Finally, these groups were also compared with regards to the patient diagnosis. As several diagnoses were noted, the groups were separated into four categories: Multinodular Goiter, Papillary Carcinoma, Follicular Adenoma, and other. The three major groups were then analyzed and compared with regards to frequency of injury and spectrum of thyroid disease.

\section{Results}

From 2005 to 2010, 230 patients were isolated who underwent a thyroidectomy with and without Intra-Operative Nerve Monitoring (IONM). While other patients were identified through the process of the chart review, they were not included in the study due to lack of appropriate documentation regarding either procedure details or post-operative notes indicating RLN function was not found. The 230 patients were separated into three categories. From 2005 to 2007, 109 patients underwent a thyroidectomy without IONM by the Otolaryngology service. From the end of 2007 to 2010, 60 patients underwent a thyroidectomy with IONM by the Otolaryngology service. A further 61 patients underwent thyroidectomy from 2005-2010 by the General Surgery service without the use of IONM.

The rates of injury within each group ranged from $5 \%$ to $7.34 \%$ (see Table 1). While the lowest rate of injury was amongst those patients who underwent a thyroidectomy with IONM, this rate was not statistically significant $(p=0.9407)$. In our study, no distinction was made between temporary and permanent injury as one of the patients in the IONM-Otolaryngology group recovered function as did two patients in the non-IONM Otolaryngology group. 171 patients were females (74.3\%), and the age ranged from 15 to 85 with an average age of 48 years.

In the IONM-Otolaryngology group, 17 patients underwent right thyroid lobectomies, 14 underwent left thyroid lobectomies, and 29 underwent total thyroidectomies. In this group, 89 nerves were at risk. Only six nerves were not identified, and of these, only one nerve was injured. The diseases included Multinodular Goiter (MNG), Follicular adenoma, Hurthle cell cancer, Papillary cancer, Follicular cancer, benign nodule, Hashimoto's thyroiditis, benign cyst, and Medullary cancer. MNG was the most common disease (see Table 2), and all three patients who suffered injury to the nerve had a diagnosis of MNG.

In the non-IONM-Otolaryngology group, 24 patients underwent right thyroid lobectomies, 31 underwent left thyroid lobectomies, and 54 underwent total thyroidectomies. In this group, 163 nerves were at risk. Nine nerves were not identified, and of these, two nerves were injured. The diseases included MNG, Follicular adenoma, Hurthle cell adenoma, Papillary cancer, Follicular cancer, benign nodule, Hashimoto's thyroiditis, benign cyst, Anaplastic cancer, Graves disease, Squamous Cell Carcinoma of the Neck with spread to the thyroid, Parathyroid cyst, Branchial Cleft Cyst, and Medullary cancer. MNG was the most common disease (see Table 3). Of the 8 patients suffering injury, 5 were diagnosed with MNG, 2 with Papillary cancer, and 1 with a Follicular adenoma.

In the non-IONM-General Surgery group, 19 patients underwent right thyroid lobectomies, 16 underwent left thyroid lobectomies, and 26 underwent total thyroidectomies. In this group, 87 nerves were at risk. Ten nerves were not identified, and of those, one nerve was injured. The diseases included MNG, Follicular adenoma, Hurthle cell adenoma, Papillary cancer, benign nodule, Hashimoto’s thyroiditis, Graves disease, and Parathyroid cancer. MNG was the most common disease (see Table 4). Of the 4 patients suffering injury, 2 were diagnosed with MNG and 2 with Papillary cancer.

Overall, 15 injuries occurred during the time span of this study, with 8 injuries occurring in the non-IONM Otolaryngology group. Of the 15 injuries, 10 patients had a diagnosis of MNG, 4 patients with Papillary cancer, and 1 patient with Follicular adenoma. 3 patients had resolution of their RLN injury while 12 were permanent.

\section{Discussion}

The most worrisome post-operative complication from a thyroidectomy is injury to the recurrent laryngeal nerve. While stretching of the nerve can occur in attempts to free the nerve of its attachments, the RLN typically recovers from this slight trauma. Direct injury to the nerve during surgical excision results in prolonged if not 
Table 1. Patient characteristics by paralysis and p-values.

\begin{tabular}{|c|c|c|c|c|c|}
\hline \multirow{2}{*}{ Variable } & & \multicolumn{3}{|c|}{ Paralysis } & \multirow{2}{*}{ Exact p-Value } \\
\hline & & No & Yes & Total & \\
\hline \multirow[t]{2}{*}{ TOTAL } & Count & 215 & 15 & 230 & Not Available \\
\hline & Percent & 93.4783 & 6.5217 & & \\
\hline \multicolumn{6}{|l|}{ GROUP } \\
\hline \multirow[t]{2}{*}{ IONM-Otolaryngology } & Count & 57 & 3 & 60 & 0.9407 \\
\hline & Percent & 95 & 5 & & \\
\hline \multirow[t]{2}{*}{ Non-IONM Otolaryngology } & Count & 101 & 8 & 109 & \\
\hline & Percent & 92.6600 & 7.3400 & & \\
\hline \multirow[t]{2}{*}{ Non-IONM Gen Surgery } & Count & 57 & 4 & 61 & \\
\hline & Percent & 93.443 & 6.5573 & & \\
\hline \multicolumn{6}{|l|}{ SEX } \\
\hline \multirow[t]{2}{*}{ Female } & Count & 161 & 10 & 171 & 0.5418 \\
\hline & Percent & 94.1500 & 5.8500 & & \\
\hline \multirow[t]{2}{*}{ Male } & Count & 54 & 5 & 59 & \\
\hline & Percent & 91.5300 & 8.4700 & & \\
\hline \multicolumn{6}{|l|}{ TYPE OF THYROIDECTOMY } \\
\hline \multirow[t]{2}{*}{ Left Lobe } & Count & 57 & 4 & 61 & 0.4608 \\
\hline & Percent & 93.4400 & 6.5600 & & \\
\hline \multirow[t]{2}{*}{ Right Lobe } & Count & 59 & 2 & 61 & \\
\hline & Percent & 96.7200 & 3.2800 & & \\
\hline \multirow[t]{2}{*}{ Total } & Count & 99 & 9 & 108 & \\
\hline & Percent & 91.6700 & 8.3300 & & \\
\hline \multicolumn{6}{|l|}{ DISEASE } \\
\hline \multirow[t]{2}{*}{ Follicular Adenoma } & Count & 38 & 1 & 39 & 0.0432 \\
\hline & Percent & 97.4400 & 2.5600 & & \\
\hline \multirow[t]{2}{*}{ MNG } & Count & 78 & 10 & 88 & \\
\hline & Percent & 88.6400 & 11.3600 & & \\
\hline \multirow[t]{2}{*}{ Other } & Count & 47 & 0 & 47 & \\
\hline & Percent & 100 & 0 & & \\
\hline \multirow[t]{2}{*}{ Papillary Carcinoma } & Count & 52 & 4 & 56 & \\
\hline & Percent & 92.8600 & 7.1400 & & \\
\hline \multirow[t]{3}{*}{ AGE } & Count & 215 & 15 & 230 & 0.8613 \\
\hline & Mean & 48.4930 & 47.8000 & 48.4478 & \\
\hline & St Deviation & 15.0046 & 11.9056 & 14.8016 & \\
\hline
\end{tabular}

Table 2. Summary of IONM-otolaryngology group.

\begin{tabular}{ccc}
\hline Thyroid Disease & Patients & Nerves Injured \\
\hline Multinodular Goiter & 23 & 3 \\
Follicular Adenoma & 12 & 0 \\
Benign Nodule & 6 & 0 \\
Hashimoto's Thyroiditis & 2 & 0 \\
Papillary Cancer & 9 & 0 \\
Follicular Cancer & 3 & 0 \\
Medullary Cancer & 1 & 0 \\
Hurthle Cell Cancer & 2 & 0 \\
Thyroid Cyst & 2 & 0 \\
\hline
\end{tabular}

Of the 60 cases, 29 total thyroidectomies, 17 right lobectomies, and 14 left lobectomies were performed. 
Table 3. Summary of non-IONM otolaryngology group.

\begin{tabular}{ccc}
\hline Thyroid Disease & Patients & Nerves Injured \\
\hline Multinodular Goiter & 42 & 5 \\
Follicular Adenoma & 16 & 1 \\
Benign Nodule & 1 & 0 \\
Hashimoto's Thyroiditis & 2 & 2 \\
Papillary Cancer & 33 & 0 \\
Anaplastic Cancer & 2 & 0 \\
Medullary Cancer & 1 & 0 \\
Hurthle Cell Adenoma & 8 & 0 \\
Parathyroid Cyst & 1 & 0 \\
Met Squamous Cell Ca & 1 & 0 \\
Branchial Cleft Cyst & 1 & 0 \\
Graves Disease & 1 & 0 \\
\hline
\end{tabular}

Of the 109 cases, 54 total thyroidectomies, 24 right lobectomies, and 31 left lobectomies were performed.

Table 4. Summary of non-IONM non-otolaryngology group.

\begin{tabular}{ccc}
\hline Thyroid Disease & Patients & Nerves Injured \\
\hline Multinodular Goiter & 23 & 2 \\
Follicular Adenoma & 11 & 0 \\
Benign Nodule & 6 & 0 \\
Hashimoto's Thyroiditis & 1 & 2 \\
Papillary Cancer & 14 & 0 \\
Graves Disease & 2 & 0 \\
Hurthle Cell Adenoma & 3 & 0 \\
\hline
\end{tabular}

Of the 61 cases, 26 total thyroidectomies, 19 right lobectomies, and 16 left lobectomies were performed.

permanent damage. With this complication in mind, identification of the recurrent laryngeal nerve during dissection of the thyroid gland is considered the gold standard in thyroidectomy [7]. Despite implementation of this step, the nerve is not easily identified or separated from the thyroid in all cases. Therefore, this complication continues to occur.

In order to further decrease the risk of injury, many Otolaryngologists have implemented intra-operative monitoring of the RLN. In addition to Otolaryngologists, General Surgeons have also adopted IONM, with both groups using IONM in about $40 \%$ - $45 \%$ of cases [9]. Despite this prevalence, the use of nerve monitoring varies across different centers [7]. A variety of nerve monitoring systems currently exist, and each serves to provide an aid to the surgeon during dissection. When there is identification and careful dissection of the nerve, the difference in rates of injury to the RLN between cases with and without IONM are minimal [3]. However, the real benefit of IONM may be one that is only realized in a small percentage of cases. The IONM study group [7] stated that the three distinct benefits of the IONM are 1) initial identification and mapping, 2) aid in dissection, and 3) immediate post-thyroid resection testing of nerve function. While the third reason provides an added benefit, it is the other two reasons which have likely increased the implementation of IONM.

There exists several RLN monitoring systems in the United States currently, but two systems have been used predominantly - Nerve Integrity Monitoring (NIM) system by Medtronic and Nerveana system by Neurovision [10]. It works through the use of an endotracheal tube with embedded vocal cord electrodes. The area where the electrodes are embedded is covered in a white tape, which is easily visible and placed just below the level of the true vocal folds by the anesthesiologist. In order to allow IONM, neuromuscular blockade cannot be employed in these patients. A hand-held probe with fine needle tip is also available for nerve mapping, identification, and 
post-resection evaluation. It is not standard to use this hand-held probe since the system provides an audible response when the RLN is manipulated or when the true vocal folds are moving.

A learning curve does exist for both the anesthesiologist and the surgeon. Appropriate placement of the embedded endotracheal tube requires some practice as the tube is more rigid than other tubes. Second, placement of the white tape on the endotracheal tube just distal to the true vocal folds is straightforward but needs to be remembered. Placing the monitor at appropriate settings can be done once, and if there is a dedicated nerve monitor, the settings will remain in place for all further thyroidectomies. The most challenging aspect with use of the nerve monitor is interpreting the audible response the monitor generates. The response may be evoked by stimulation of the RLN by the surgeon or if the patient begins to awaken. It is important to discern this difference especially when the surgeon has started to dissect and isolate the RLN.

Several studies have been conducted demonstrating that differences between procedures conducted with and without IONM is minimal with regards to RLN injury. Within this study, there is a slight decrease in the number of cases in which the RLN was injured following implementation of IONM. The overall percentage of injuries was $5 \%$ in the IONM-Otolaryngology group, which was less than the $7.34 \%$ noted in the Non-IONM Otolaryngology group. However, the $\mathrm{p}$-value $(\mathrm{p}=0.9407)$ was not significant. These rates of injury fall within the range seen in several studies analyzing frequency of RLN injury following thyroid surgery, $1.1 \%-12.5 \%$ with IONM and $2.6 \%-10.1 \%$ without IONM [3] [11]-[15]. Interestingly, two small studies reviewed showed decreased rates of RLN injury without the IONM [13] [16]. Further, the largest study reviewed, performed by Dralle, reviewed greater than 22,000 nerves at risk and identified a rate of $2.7 \%$ injury with IONM and $2.6 \%$ without IONM [3]. Therefore, the overall benefit of IONM remains equivocal.

An important observation lies in the diagnosis of those patients with injuries. Including all three groups, 15 patients in this study were injured with 10 patients having a diagnosis of Multinodular Goiter. Further, all 3 patients injured with use of the IONM had a diagnosis of Multinodular Goiter. As our study encompasses only a group of 215 patients, a larger study would be needed to see if this observation persists. This finding suggests that the most difficult of thyroid dissection may be in the case of Multinodular goiter. While this statement seems to bear truth anecdotally, a larger study correlating injury rates with patient diagnosis is needed to confirm this observation.

In this study, all nerve injuries occurred in patients with three diagnoses: Multinodular Goiter (10), Papillary Carcinoma (4), and Follicular Adenoma (1). Despite a small patient sample, Multinodular Goiter and Papillary Cancer are the most common reason for thyroidectomy and were observed to be most common in this study. It also begs the question if the use of the nerve monitor system should be based on a patient's diagnosis. Recent studies that include the diagnosis of the patient reinforce the idea that the most common diagnosis for patients undergoing thyroidectomy is Multinodular Goiter along with Thyroid Cancer, of which Papillary is the most common [16]. A possible reason for increased RLN injury in Multinodular Goiter may be that the growth of the thyroid causes the RLN to take a more tortuous route. The preliminary information from this study suggests that in these cases the benefit of IONM may be the greatest, especially if used to locate and even trace the nerve. Other factors, such as a previously operated patient or a patient who has undergone radiation, may also cause changes to standard anatomy making isolation of the RLN challenging. Implementation of IONM in these cases may also prove beneficial to the surgeon.

\section{Conclusion}

Further studies analyzing the use of IONM including the diagnosis of patients are merited, it appears that the benefit of IONM is minimal. When an experienced surgeon performs the thyroidectomy and isolates the RLN, there is minimal risk for injury. Appropriate usage of IONM by acknowledging its benefits with regard to identification or aid in dissection can help in challenging cases. However, the surgeon must recognize that nerve injury can occur without visual identification of the nerve. The cases in which IONM provides the greatest benefit are likely those in which anatomy is obscured, possibly most often in the case of MNG. Also, a nerve monitoring system can be easily incorporated into practice although it does possess a minimal learning curve for both the anesthesiologist and surgeon. With its simplicity, along with the uncertainty of each patient's RLN anatomy, the surgeon stands to gain from implementation of IONM. On the other hand, anomalous or tortuous paths of RLN cannot be predicted. Implementing the system does increase the cost of the procedure. While operating room time and post-operative hospital stays contribute more to the overall cost of the procedure, the cost of the system is not insignificant. Thus, the final decision lies in the hands of the surgeon and his/her level of expe- 
rience with performing thyroidectomies. Most important, it cannot be stressed enough that IONM is only a supplement to guide the surgeon in identification of the RLN, the only proven method to prevent permanent injury.

\section{References}

[1] Lahey, F.H. (1932) Routine Dissection and Demonstration of the Recurrent Laryngeal Nerve in Subtotal Thyroidectomy. Surgery Gynecology \& Obstetrics, 66, 775-777.

[2] Riddell, V.H. (1956) Injury to Recurrent Laryngeal Nerves during Thyroidectomy-A Comparison between the Results of Identification and Non-Identification in 1022 Nerves Exposed to Risk. The Lancet, 29, 638-641. http://dx.doi.org/10.1016/S0140-6736(56)92333-9

[3] Dralle, H., Sekulla, C., Haerting, J., et al. (2004) Risk Factors of Paralysis and Functional Outcome after Recurrent Laryngeal Nerve Monitoring in Thyroid Surgery. Surgery, 136, 1310-1322. http://dx.doi.org/10.1016/j.surg.2004.07.018

[4] Randolph, G.W. (2003) Surgical Anatomy of the Recurrent Laryngeal Nerve. In: Randolph, G.W., Ed., Surgery of the Thyroid and Parathyroid Glands, W.B. Saunders, Philadelphia.

[5] Snyder, S.K. and Hendricks, J.C. (2005) Intraoperative Neurophysiology Testing of the Recurrent Laryngeal Nerve: Plaudits and Pitfalls. Surgery, 138, 1183-1192. http://dx.doi.org/10.1016/j.surg.2005.08.027

[6] Hermann, M., Alk, G., Roka, R., et al. (2002) Laryngeal Recurrent Nerve Injury in Surgery for Benign Thyroid Diseases-Effect of Nerve Dissection and Impact of Individual Surgeon in More than 27,000 Nerves at Risk. Annals of Surgery, 235, 261-268. http://dx.doi.org/10.1097/00000658-200202000-00015

[7] Randolph, G.W., Dralle, H., et al. (2011) Electrophysiologic Recurrent Laryngeal Nerve Monitoring during Thyroid and Parathyroid Surgery: International Standards Guideline Statement. Laryngoscope, 121, S1-S16. http://dx.doi.org/10.1002/lary.21119

[8] Barczynski, M., Konturek, A. and Cichon, S. (2009) Randomized Clinical Trial of Visualization versus Neuromonitoning of Recurrent Laryngeal Nerves during Thyroidectomy. British Journal of Surgery, 96, 240-246. http://dx.doi.org/10.1002/bjs.6417

[9] Horne, S.K., Gal, T.J. and Brennan, J.A. (2007) Prevalence and Patterns of Intraoperative Nerve Monitoring for Thyroidectomy. Otolaryngology_Head and Neck Surgery, 136, 952-956. http://dx.doi.org/10.1016/j.otohns.2007.02.011

[10] Angelos, P. (2009) Recurrent Laryngeal Nerve Monitoring: State of the Art, Ethical and Legal Issues. Surgical Clinics of North America, 89, 1157-1169.

[11] Brauckhoff, M., Gimm, O. and Thanh, P.N. (2002) First Experiences in Intraoperative Neurostimulation of the Recurrent Laryngeal Nerve during Thyroid Surgery of Children and Adolescents. Journal of Pediatric Surgery, 37, 14141418. http://dx.doi.org/10.1053/jpsu.2002.35403

[12] Yarbrough, D.E., Thompson, G.B., Kasperbauer, J.L., et al. (2004) Intraoperative Electromyographic Monitoring of the Recurrent Laryngeal Nerve in Reoperative Thyroid and Parathyroid Surgery. Surgery, 136, 1107-1115. http://dx.doi.org/10.1016/j.surg.2004.06.040

[13] Robertson, M.L., Steward, D.L., Gluckman, J.L., et al. (2004) Continuous Laryngeal Nerve Integrity Monitoring during Thyroidectomy: Does It Reduce Risk of Injury? Otolaryngology—Head and Neck Surgery, 131, 596-600. http://dx.doi.org/10.1016/j.otohns.2004.05.030

[14] Chan, W.F., Lang, B.H.H. and Lo, C.Y. (2006) The Role of Intraoperative Neuromonitoring of Recurrent Laryngeal Nerve during Thyroidectomy: A Comparative Study on 1000 Nerves at Risk. Surgery, 140, 866-873. http://dx.doi.org/10.1016/j.surg.2006.07.017

[15] Shindo, M. and Chheda, N.N. (2007) Incidence of Vocal Cord Paralysis with and without Recurrent Laryngeal Nerve Monitoring during Thyroidectomy. Archives of Otolaryngology—Head and Neck Surgery, 133, 481-485. http://dx.doi.org/10.1001/archotol.133.5.481

[16] Duclos, A., Lifante, J.C., Ducaroz, S., Soardo, P., Colin, C. and Peix, J.L. (2011) Influence of Intraoperative Neuromonitoring on Surgeons' Technique during Thyroidectomy. World Journal of Surgery, 35, 773-778.

http://dx.doi.org/10.1007/s00268-011-0963-4 
Scientific Research Publishing (SCIRP) is one of the largest Open Access journal publishers. It is currently publishing more than 200 open access, online, peer-reviewed journals covering a wide range of academic disciplines. SCIRP serves the worldwide academic communities and contributes to the progress and application of science with its publication.

Other selected journals from SCIRP are listed as below. Submit your manuscript to us via either submit@scirp.org or Online Submission Portal.
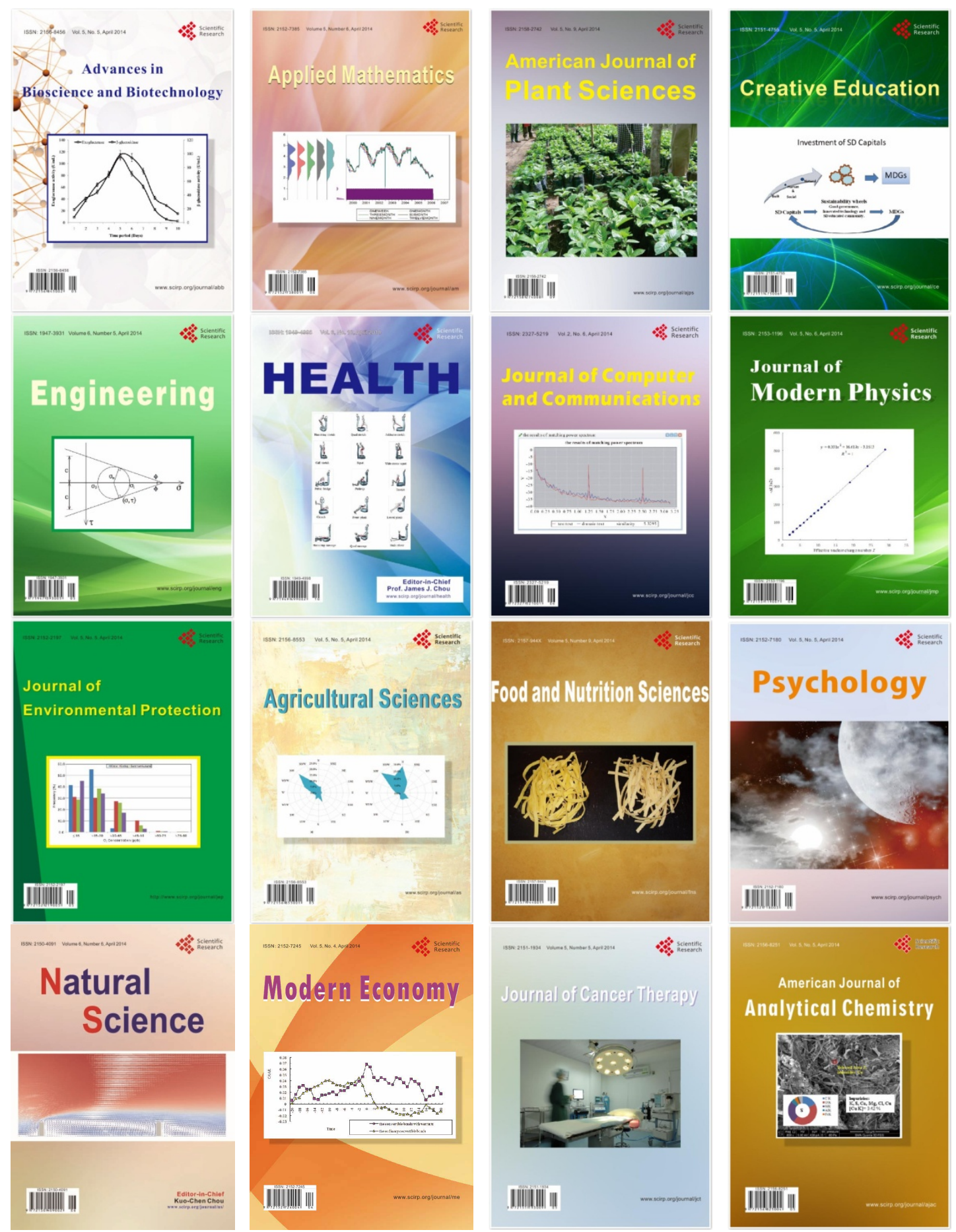